Olivier Glass (Paris)

Piotr BogusŁaw Mucha (Warszawa)

\title{
INVISCID LIMIT FOR THE 2-D STATIONARY EULER SYSTEM WITH ARBITRARY FORCE IN SIMPLY CONNECTED DOMAINS
}

Abstract. We study the convergence in the vanishing viscosity limit of the stationary incompressible Navier-Stokes equation towards the stationary Euler equation, in the presence of an arbitrary force term. This requires that the fluid is allowed to pass through some open part of the boundary.

1. Introduction. We investigate the steady Navier-Stokes equations in a two-dimensional bounded simply connected domain $\Omega$ with a smooth boundary $\partial \Omega$, with an external force term $F$. The system reads

$$
\begin{array}{ll}
v \cdot \nabla v-\nu \Delta v+\nabla p=F & \text { in } \Omega, \\
\operatorname{div} v=0 & \text { in } \Omega,
\end{array}
$$

where $v=\left(v^{1}, v^{2}\right)$ represents the velocity of the fluid, $p$ its pressure, and $\nu$ is the constant positive viscosity coefficient.

More precisely, we will be interested in the inviscid limit towards the Euler equation. Let us recall that for the stationary Euler system with homogeneous boundary conditions ( $\vec{n}$ the unit outward normal vector to $\partial \Omega$ ) and a force term:

$$
\begin{array}{ll}
v \cdot \nabla v+\nabla p=F & \text { in } \Omega, \\
\operatorname{div} v=0 & \text { in } \Omega, \\
v \cdot \vec{n}=0 & \text { on } \partial \Omega,
\end{array}
$$

2000 Mathematics Subject Classification: 76D09, 76D03, 35Q30, 76 B03.

Key words and phrases: inviscid limit, stationary flow, Navier-Stokes equations, Euler system. 
there is no solution in general. Typically, integrating (1.2) on $\partial \Omega$ in the direction of the tangent $(\vec{\tau}$ the tangent vector to $\partial \Omega)$, we would get

$$
\int_{\partial \Omega} F \cdot \vec{\tau} d \sigma=0,
$$

which is not satisfied by any $F$ (this is Kelvin's law for the stationary Euler equation). Moreover, even if we restrict ourselves to $F$ satisfying (1.3), there might be no solution to (1.2). For instance, consider $F$ satisfying (1.3) and $\operatorname{rot} F>0$ on $\partial \Omega$. Writing (1.2) in vorticity form:

$$
v \cdot \nabla \alpha=\operatorname{rot} F \quad \text { in } \Omega,
$$

where the vorticity is defined by

$$
\alpha=\operatorname{rot} v=\partial_{x_{1}} v^{2}-\partial_{x_{2}} v^{1},
$$

we see by using characteristics that one cannot define $\alpha$ completely on the boundary. On the other hand, it was shown by Coron [3] that if one allows the fluid to pass through an arbitrarily small part $\Gamma$ of the boundary, then the Euler system has a solution for any $F$ (in the case of a simply connected domain; see [5] in the general case).

Hence we investigate the Navier-Stokes equation with boundary conditions that will be allowed to be nonhomogeneous on an arbitrary part of the boundary. Let $\Gamma$ be an arbitrary nonempty open part of $\partial \Omega$, which will represent the zone where we can put nonhomogeneous conditions. We will consider equation (1.1) supplied with the following boundary condition on $\partial \Omega \backslash \Gamma$ :

$$
\begin{array}{ll}
v \cdot \vec{n}=0 & \text { on } \partial \Omega \backslash \Gamma, \\
\frac{\partial \alpha}{\partial \vec{n}}=0 & \text { on } \partial \Omega \backslash \Gamma .
\end{array}
$$

Hence the question we raise is the following: given $\Omega$ and $\Gamma$, for any $F$, can we find a solution of (1.1)-(1.4) for all suitably small $\nu$ ?

Let us mention that this problem is connected to control theory. It is indeed known that for finite-dimensional control systems, the stabilizability property involves the existence of stationary solutions for small right hand side (see Brockett [1]). A similar phenomenon is observed for the boundary stabilizability of the incompressible Euler equation and raises the same question for the Navier-Stokes equation. See $[3,4,6]$ for more details. The evolutionary Euler system with inflow conditions has been studied in $[12-14]$. 
Solutions to (1.1), (1.4) will be found by solving the following system:

$$
\begin{array}{ll}
v \cdot \nabla \alpha-\nu \Delta \alpha=\operatorname{rot} F & \text { in } \Omega, \\
\operatorname{rot} v=\alpha & \text { in } \Omega, \\
\operatorname{div} v=0 & \text { in } \Omega, \\
v \cdot \vec{n}=d & \text { on } \partial \Omega, \\
\alpha=\alpha_{\text {in }} & \text { on } \Gamma_{\text {in }}, \\
\partial \alpha / \partial \vec{n}=0 & \text { on } \partial \Omega \backslash \Gamma_{\text {in }} .
\end{array}
$$

Let us emphasize that thanks to the simple connectedness of the domain $\Omega$ the system $(1.5)_{1,2,3}$ is completely equivalent to the original equation (1.1). The choice of the boundary condition $(1.5)_{6}$ deserves a comment. Since we are going examine the inviscid limit of solutions to (1.5) this relation should disappear in the limit. Additionally, the inhomogeneous data $(1.5)_{4,5}$ are required to be preserved, to control the well posedness of the limit Euler system. In a sense, we may look at (1.5) as a regularization of the system (1.2), thus we can find an analogy to considerations for the continuity equation in the theory of weak solutions to the compressible Navier-Stokes equations [9].

In the above equation, one will choose $d$ suitably; in particular, one requires

$$
\operatorname{supp} d \subset \Gamma \text { and } \quad \int_{\partial \Omega} d d \sigma=0
$$

in order that $(1.4)_{4}$ be satisfied. Also we introduce

$$
\Gamma_{\mathrm{in}}=\{x \in \Gamma \mid d(x)<0\} \quad \text { and } \quad \Gamma_{\text {out }}=\{x \in \Gamma \mid d(x)>0\} .
$$

Our first aim is the following result.

TheOREM 1. For any $(\Omega, \Gamma)$, there exists $\bar{d} \in C^{\infty}(\Gamma)$ such that the following holds. Let $F \in H^{1}\left(\Omega ; \mathbb{R}^{2}\right)$ and $\alpha_{\mathrm{in}} \in H^{3 / 2}\left(\Gamma_{\mathrm{in}} ; \mathbb{R}\right)$, where $\Gamma_{\mathrm{in}}$ is defined in (1.7) with $d=\bar{d}$. Then there exists $\bar{l}$ such that for $0<\nu \leq 1$, for any $l>\bar{l}$ there exists at least one weak solution to the system (1.5) with

$$
d(x)=l \bar{d}(x),
$$

such that $v \in C^{a}(\bar{\Omega})$ with $a<1 / 2$ and

$$
\|\operatorname{rot} v\|_{L_{2}(\Omega)}+\|v\|_{C^{a}(\bar{\Omega})} \leq C(\mathrm{DATA}, l),
$$

where the r.h.s. of (1.9) is independent of $\nu$.

The main difficulty is to obtain the estimate (1.9), giving relatively high regularity of solutions with no dependence on the viscosity coefficient $\nu$. The solutions will be constructed as perturbations of a given potential flow, which is related to the function $\bar{d}$ defined on $\Gamma$. Such a flow will be constructed in 
the next section. Our technique is based on the energy approach, but used in a nonstandard way. For chosen $d$ in (1.8) the solution to (1.5) is unique, by the Banach fixed point theorem. However, the $d$ is constructed in the proof, so this feature is not emphasized in the statement of the theorem. The bound (1.9) is a motivation for the next result.

Our second aim is to analyze the inviscid limit of solutions given by Theorem 1.

THEOREM 2. Under the assumptions of Theorem 1, let $v^{\nu}$ denote the solution to (1.5) with viscosity $\nu$. Then there exists $v^{E} \in C^{1 / 2-\delta}(\bar{\Omega})$ with $\delta>0$ such that for a subsequence $\nu_{k} \rightarrow 0^{+}$as $k \rightarrow \infty$,

$$
v^{\nu_{k}} \rightarrow v^{E} \quad \text { in } C^{1 / 2-\delta}(\bar{\Omega}) \quad \text { and } \quad v^{\nu_{k}} \rightarrow v^{E} \quad \text { in } H^{1}(\Omega)
$$

for $k \rightarrow \infty$ and $v^{E}$ satisfies the Euler system

$$
\begin{array}{ll}
v^{E} \cdot \nabla v^{E}+\nabla p^{E}=F & \text { in } \Omega, \\
\operatorname{div} v^{E}=0 & \text { in } \Omega, \\
v^{E} \cdot \vec{n}=d & \text { on } \partial \Omega, \\
\operatorname{rot} v^{E}=\alpha_{\text {in }} & \text { on } \Gamma_{\mathrm{in}},
\end{array}
$$

where $p^{E}$ is a constructible pressure.

The main element in the proof of Theorem 2 is the a priori estimate (1.9). In the evolutionary case the basic bound follows from the energy estimate, but in the stationary case we lose this possibility. The information given by Theorem 1 is not sufficient. We need more sophisticated estimates for higher derivatives of solutions in terms of dependence on $\nu$. This will allow us to control the dependence on the boundary condition $\alpha_{\text {in }}$, which could be omitted in straightforward considerations. The meaning of the solution to (1.11) will be defined later by (4.10). The resulting regularity and weak formulation will allow us to control the dependence on the boundary da$\operatorname{tum} \alpha_{\text {in }}$.

There are no general results concerning the inviscid limit of the stationary Navier-Stokes system towards the stationary Euler system; the only known results have been proved in [7], [8], but for the unforced system in a special type of domain.

Just as for Theorem 1, fixing $d$ we are able to obtain the uniqueness of the limit, so (1.10) holds for an arbitrary sequence, not only for a subsequence. Additionally in that case (1.11) admits unique solutions and it is again a consequence of the fixed point approach in the proof of (1.9). We omit these considerations in the proofs, since this property holds for our particular $d$ and is not proven for general data. 
The structure of the paper is the following. In Section 2, we introduce the function $\bar{d}$ announced in Theorem 1; in Section 3, we establish Theorem 1; in Section 4, we establish Theorem 2; finally, Section 5 is an Appendix where we have put technical yet central results.

Throughout the paper we use standard notation. The letter $C$ denotes a generic constant independent of $\nu$ and $\lambda$; DATA depends on norms of data and known quantities and is independent of $\nu$ and $\lambda$.

2. A proposition. In this section we introduce a function $\theta$ on which the construction depends. This is given in the next proposition:

Proposition 2.1. Let $\Omega$ be a bounded smooth simply connected domain in $\mathbb{R}^{2}$, and let $\Gamma$ be a nonempty open part of $\partial \Omega$. Then there exists $\theta \in$ $C^{\infty}(\bar{\Omega} ; \mathbb{R})$ such that

$$
\begin{gathered}
\Delta \theta=0 \quad \text { in } \Omega, \quad|\nabla \theta| \geq k>0 \quad \text { in } \bar{\Omega} \\
\nabla \theta \cdot \vec{n}=0 \quad \text { on } \partial \Omega \backslash \Gamma, \\
\forall t, \quad\{x \in \bar{\Omega} \mid \theta(x) \leq t\} \text { is a piecewise smooth domain. }
\end{gathered}
$$

REMARK 2.1. For the rest of the paper, we fix

$$
\bar{d}:=\nabla \theta \cdot \vec{n} \quad \text { on } \partial \Omega,
$$

and as we will consider boundary conditions $(1.5)_{4}$ of the type $d=l \bar{d}$, we will always have, according to the definition (1.7),

$$
\begin{gathered}
\Gamma_{\text {in }}=\{x \in \partial \Omega: \nabla \theta(x) \cdot \vec{n}(x)<0\}=\{x \in \partial \Omega: d(x)<0\}, \\
\Gamma_{\text {out }}=\{x \in \partial \Omega: \nabla \theta(x) \cdot \vec{n}(x)>0\}=\{x \in \partial \Omega: d(x)>0\} .
\end{gathered}
$$

Shrinking $\Gamma$ if necessary, we suppose from now on that $\Gamma=\Gamma_{\text {in }} \cup \Gamma_{\text {out }}$.

Proof of Proposition 2.1. The statement without condition (2.3) was established in [2]. Here we proceed as follows. Consider the square $[0,1]^{2}$ in $\mathbb{R}^{2}$. Extend it inside the strip $\mathbb{R} \times[0,1]$ to a smooth bounded contractible domain $U$. Now it follows from Riemann's mapping theorem that $U$ and $\Omega$ are conformally equivalent. Moreover, it is a standard result that the corresponding mapping is $C^{\infty}$ up to the boundary, as follows from the smoothness of $\Omega$ and $U$ (see for instance [10]).

Now a conformal map of a simply connected domain is defined up to the conformal group of the disc, which is the following 3-parameter group:

$$
G=\left\{g(z)=e^{i \phi} \frac{a+z}{1+\bar{a} z}|a \in \mathbb{C},| a \mid<1, \phi \in \mathbb{R}\right\}
$$


Hence one can specify a unique conformal mapping $\varphi$ between $U$ and $\Omega$ by fixing the image of three points of the boundary of $U$ in the boundary of $\Omega$. We proceed as described in Figure 1. Precisely, shrinking $\Gamma$ if necessary, we can suppose that it is connected. Let $\{\widetilde{A}, \widetilde{B}\}$ be its boundary inside $\partial \Omega$. Set $A:=(1,0), B=(1,1)$ and $C=(0,1 / 2)$. We choose the conformal map $\varphi$ from $U$ to $\Omega$ so that it sends $A$ to $\widetilde{A}, B$ to $\widetilde{B}$, and $C$ inside $\Gamma$.
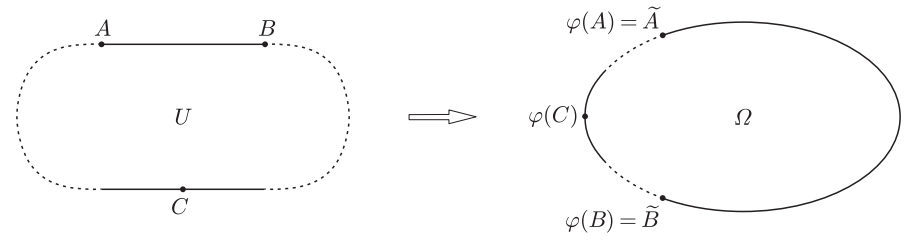

Fig. 1. Conformal map

We recall that on simply connected domains, there is an equivalence between holomorphic functions and gradients of harmonic functions via the following rule:

$$
\begin{aligned}
& \psi=\psi^{1}+i \psi^{2} \text { is holomorphic in } \Omega \\
& \Leftrightarrow\left(\psi^{1},-\psi^{2}\right) \text { is the gradient of a harmonic function. }
\end{aligned}
$$

Now we consider the map $\theta$ obtained by transporting onto $\Omega$ the harmonic map $\left(x_{1}, x_{2}\right) \mapsto x_{1}$ defined on $U$ through $\varphi, \theta(\varphi(x))=x$ on $U$. For this $\theta$, properties (2.1) and (2.2) come from the conformality and the fact that $(1,0)$ is tangent to the part of the boundary $\partial U$ given by $[A, B]$. Finally, (2.3) comes directly from the fact that

$$
\left\{x=\left(x_{1}, x_{2}\right) \in U \mid x_{1} \leq t\right\} \text { is a piecewise smooth domain. }
$$

Proposition 2.1 is proved.

3. Proof of Theorem 1. In this section we prove Theorem 1. The proof is divided into two parts. First, we restrict our attention to sufficiently small data, but the viscosity coefficient is not restricted, i.e. it can be arbitrarily small. Next, we consider the general case by using a homogeneity argument.

Let us consider the case of small data. We look for solutions in the vicinity of the potential flow $\nabla \theta$ constructed in Proposition 2.1.

Let the solution to (1.5) be considered in the following form:

$$
v=\nabla \theta+u \quad \text { and put } \quad v \cdot \vec{n}=\nabla \theta \cdot \vec{n}=: \bar{d} \quad \text { on } \partial \Omega .
$$


Then the system (1.5) in terms of $u$ takes the form

$$
\begin{array}{ll}
\nabla \theta \cdot \nabla \alpha-\nu \Delta \alpha=\operatorname{rot} F-u \cdot \nabla \alpha & \text { in } \Omega, \\
\operatorname{rot} u=\alpha & \text { in } \Omega, \\
\operatorname{div} u=0 & \text { in } \Omega, \\
u \cdot \vec{n}=0 & \text { on } \partial \Omega, \\
\alpha=\alpha_{\text {in }} & \text { on } \Gamma_{\text {in }}, \\
\partial \alpha / \partial \vec{n}=0 & \text { on } \partial \Omega \backslash \Gamma_{\text {in }} .
\end{array}
$$

Our technique requires a special parameterization of the domain $\Omega$. Thanks to Proposition 2.1 we can use the properties of the function $\theta$. The set $\Omega$ is parameterized by a set $D \subset \mathbb{R}^{2}$ in such a way that

$$
Y_{t}=\{x \in \Omega: \theta(x)=t\},
$$

$Y_{t}$ is parameterized by a coordinate $s \in \mathbb{R}$, and we have a diffeomorphism

$$
D \ni(s, t) \leftrightarrow x \in \Omega .
$$

Precisely, one chooses $D$ to be the $U$ of the proof of Proposition 2.1. Additionally, we introduce

$$
X_{t}=\bigcup_{t^{\prime}<t} Y_{t^{\prime}}=\{x \in \Omega: \theta(x)<t\}
$$

for $t \in\left(t_{\min }, t_{\max }\right)$ and $X_{t_{\max }}=\Omega$ and $X_{t_{\min }}=\emptyset$.

Let us remark that $\partial X_{t}$ can be divided into several parts: $Y_{t}$ and $\partial X_{t} \cap \partial \Omega$, which can itself be divided into $\partial X_{t} \cap \Gamma_{\mathrm{in}}, \partial X_{t} \cap(\partial \Omega \backslash \Gamma)$ and $\partial X_{t} \cap \Gamma_{\text {out }}$ (which is not empty for large $t$ ). Clearly, this decomposition is trivial when transported to $U$.

The proof of existence of solutions to (3.2) will rely on the Banach fixed point theorem. First, we prove an a priori estimate, describing the function spaces for the solutions.

A priori estimate. To start our estimation we have to find an extension of the boundary vorticity. We easily find $\widetilde{\alpha} \in H^{2}(\Omega)$ such that

$$
\|\widetilde{\alpha}\|_{H^{2}(\Omega)} \leq C\left\|\alpha_{\mathrm{in}}\right\|_{H^{3 / 2}\left(\Gamma_{\mathrm{in}}\right)},\left.\quad \widetilde{\alpha}\right|_{\Gamma_{\mathrm{in}}}=\alpha_{\mathrm{in}},\left.\quad \widetilde{\alpha}\right|_{\Gamma_{\text {out }}}=0 .
$$

Additionally, we choose $\widetilde{\alpha}$ to be a harmonic function:

$$
\Delta \widetilde{\alpha}=0 \quad \text { in } \Omega .
$$

Multiplying $(3.2)_{1}$ by $\alpha-\widetilde{\alpha}$ and integrating over $X_{t}$ we get

$$
\begin{aligned}
\int_{X_{t}}[\nabla \theta \cdot \nabla \alpha(\alpha-\widetilde{\alpha})-\nu \Delta \alpha & (\alpha-\widetilde{\alpha})] d x \\
& =\int_{X_{t}}[\operatorname{rot} F(\alpha-\widetilde{\alpha})+u \nabla \alpha(\alpha-\widetilde{\alpha})] d x .
\end{aligned}
$$


The first term on the 1.h.s. of (3.5) reads

$$
\begin{aligned}
\frac{1}{2} \int_{X_{t}} \nabla \theta \cdot \nabla \alpha^{2} d x & -\int_{X_{t}} \nabla \theta \cdot \nabla \alpha \widetilde{\alpha} d x \\
= & \frac{1}{2} \int_{Y_{t}}|\nabla \theta| \alpha^{2} d \sigma+\int_{X_{t}} \nabla \theta \cdot \nabla \widetilde{\alpha} \alpha d x-\int_{Y_{t}}|\nabla \theta| \alpha \widetilde{\alpha} d \sigma \\
& -\frac{1}{2} \int_{\partial X_{t} \cap \Gamma_{\text {in }}} \nabla \theta \cdot \vec{n} \alpha_{\text {in }}^{2} d \sigma+\frac{1}{2} \int_{\partial X_{t} \cap \Gamma_{\text {out }}} \nabla \theta \cdot \vec{n} \alpha^{2} d \sigma,
\end{aligned}
$$

where we used the fact $\nabla \theta \cdot \vec{n}=|\nabla \theta|$ on $Y_{t}$ and $u \cdot \vec{n}=0$ on $\partial \Omega \backslash \Gamma$. Now we remark that by (2.4) the integral over $\partial X_{t} \cap \Gamma_{\text {out }}$ is nonnegative; hence we can forget this term and obtain a lower bound for the first term on the 1.h.s. of (3.5).

The second term takes the form

$$
\begin{aligned}
-\nu \int_{X_{t}} \Delta \alpha(\alpha-\widetilde{\alpha}) d x= & \nu \int_{X_{t}}|\nabla \alpha|^{2} d x-\nu \int_{X_{t}} \nabla \alpha \cdot \nabla \widetilde{\alpha} d x \\
& -\nu \int_{Y_{t}} \frac{\partial \alpha}{\partial \vec{n}} \alpha d \sigma+\nu \int_{Y_{t}} \frac{\partial \alpha}{\partial \vec{n}} \widetilde{\alpha} d \sigma .
\end{aligned}
$$

The remaining boundary terms vanish by $(3.2)_{5,6}$ and (3.3).

The last term of the r.h.s. of (3.5) has the form

$$
\begin{aligned}
\int_{X_{t}} u \cdot \nabla \alpha \alpha d x-\int_{X_{t}} u \cdot \nabla \alpha \widetilde{\alpha} d x \\
=\frac{1}{2} \int_{Y_{t}} \vec{n} \cdot u \alpha^{2} d \sigma+\int_{X_{t}} u \cdot \nabla \widetilde{\alpha} \alpha d x-\int_{Y_{t}} \vec{n} \cdot u \alpha \widetilde{\alpha} d \sigma .
\end{aligned}
$$

Here the remaining boundary terms vanish thanks to (3.3) and (3.2) 4 .

Applying the standard Schwarz inequality we infer that for some constant $C$ independent of $\nu$,

$$
\left|\nu \int_{Y_{t}} \frac{\partial \alpha}{\partial n} \alpha d \sigma\right| \leq \frac{k}{4} \sup _{t} \int_{Y_{t}} \alpha^{2} d \sigma+C \sup _{t} \nu^{2} \int_{Y_{t}}|\nabla \alpha|^{2} d \sigma .
$$

In the above estimate, the constant $k$ is given by (2.1).

We conclude that

$$
\begin{aligned}
& \frac{k}{4} \sup _{t} \int_{Y_{t}} \alpha^{2} d \sigma+\nu \int_{\Omega}|\nabla \alpha|^{2} d x \\
& \leq\|u\|_{L_{\infty}(\Omega)} \sup _{t} \int_{Y_{t}} \alpha^{2} d \sigma+C \sup _{t} \nu^{2} \int_{Y_{t}}|\nabla \alpha|^{2} d \sigma \\
& \quad+C \sup _{t} \int_{Y_{t}} \widetilde{\alpha}^{2} d \sigma+\int_{\Omega}|\nabla \widetilde{\alpha}|^{2} d x+\int_{\Omega}(\operatorname{rot} F)^{2} d x+C \int_{\Gamma_{\mathrm{in}}} \alpha_{\mathrm{in}}^{2} d \sigma .
\end{aligned}
$$


The main difficulty is the term containing the trace of the gradient of $\alpha$ on $Y_{t}$, the second term on the r.h.s. of (3.6). To estimate it we have to apply the trace theorem for the Besov space $B_{2,1}^{1 / 2}(\Omega)$. In this critical case, the trace is still controlled since $Y_{t}$ is a smooth submanifold, i.e. for a sufficiently regular function $f$ we have

$$
\left\|\left.f\right|_{Y_{t}}\right\|_{L_{2}\left(Y_{t}\right)} \leq C\|f\|_{B_{2,1}^{1 / 2}(\Omega)} \quad \text { for all } t \in\left(t_{\min }, t_{\max }\right) .
$$

The definition of this space is quite complex, but it can be represented as an interpolation space

$$
B_{2,1}^{1 / 2}(\Omega)=\left(H^{1}(\Omega), L_{2}(\Omega)\right)_{1 / 2,1}
$$

(see [11]); this guarantees the estimate

$$
\|f\|_{B_{2,1}^{1 / 2}(\Omega)} \leq C\|f\|_{H^{1}(\Omega)}^{1 / 2}\|f\|_{L_{2}(\Omega)}^{1 / 2} .
$$

Thus from (3.7) and (3.8) we easily conclude that the boundary term can be estimated as follows:

$$
\nu^{2} \int_{Y_{t}}|\nabla \alpha|^{2} d \sigma \leq C \nu^{2}\|\nabla \alpha\|_{L_{2}(\Omega)}\left(\left\|\nabla^{2} \alpha\right\|_{L_{2}(\Omega)}+\|\nabla \alpha\|_{L_{2}(\Omega)}\right) .
$$

To make use of the above inequality we need to control the second gradient of $\alpha$ in terms of $\nu$. We will apply the following result, which we prove in the Appendix.

Theorem 3. Let $G \in L_{2}(\Omega), \theta \in W_{\infty}^{1}(\Omega)$, and assume that $\lambda$, a parameter of the localization defined by (5.2), is sufficiently small. Then solutions to

$$
\begin{array}{ll}
\nabla \theta \cdot \nabla \beta-\nu \Delta \beta=G & \text { in } \Omega, \\
\beta=\beta_{\text {in }} & \text { on } \Gamma_{\mathrm{in}}, \\
\partial \beta / \partial \vec{n}=0 & \text { on } \partial \Omega \backslash \Gamma_{\mathrm{in}}
\end{array}
$$

satisfy the following bound (for $0<\lambda<\lambda_{0}(\partial \Omega)$ ):

$$
\begin{array}{r}
\nu\left\|\nabla^{2} \beta\right\|_{L_{2}(\Omega)} \leq C\left[\left(\nu \lambda^{-1}+\lambda\right)\|\nabla \beta\|_{L_{2}(\Omega)}+\left(\nu \lambda^{-2}+1\right)\|\beta\|_{L_{2}(\Omega)}\right. \\
\left.+\|G\|_{L_{2}(\Omega)}+\left\|\beta_{\text {in }}\right\|_{H^{3 / 2}\left(\Gamma_{\text {in }}\right)}\right] .
\end{array}
$$

Applying Theorem 3 to estimate $\nu\left\|\nabla^{2} \alpha\right\|_{L_{2}(\Omega)}$ (and using (3.2) $)_{1}$ ) we conclude that

$$
\begin{array}{r}
\nu^{2} \int_{Y_{t}}|\nabla \alpha|^{2} d \sigma \leq C \nu\|\nabla \alpha\|_{L_{2}(\Omega)}\left(\|\nabla \alpha\|_{L_{2}(\Omega)}\left(\nu \lambda^{-1}+\lambda+\nu+\|u\|_{L_{\infty}(\Omega)}\right)\right. \\
\left.+\|\alpha\|_{L_{2}(\Omega)}\left(\nu \lambda^{-2}+1\right)+\|\operatorname{rot} F\|_{L_{2}(\Omega)}+\left\|\alpha_{\text {in }}\right\|_{H^{3 / 2}\left(\Gamma_{\text {in }}\right)}\right) .
\end{array}
$$

Now, we restrict ourselves to the case

(3.13) $\quad \nu \leq \lambda^{2} \leq 1$ and hence $\nu \lambda^{-1}+\lambda+\nu \leq 3 \lambda$ and $\nu \lambda^{-2}+1 \leq 2$.

This can be obtained for any $\lambda>0$ by choosing $\nu$ sufficiently small. 
Using $\nu\|\nabla \alpha\|_{L^{2}(\Omega)}\|\alpha\|_{L^{2}(\Omega)} \leq \nu^{2} \lambda^{-1}\|\nabla \alpha\|_{L^{2}(\Omega)}^{2}+\lambda\|\alpha\|_{L^{2}(\Omega)}^{2}$ and taking (3.13) into account we deduce that

$$
\begin{aligned}
\nu^{2} \int_{Y_{t}}|\nabla \alpha|^{2} d \sigma \leq & C \nu\left(3 \lambda+\|u\|_{L_{\infty}(\Omega)}\right)\|\nabla \alpha\|_{L_{2}(\Omega)}^{2} \\
& +C \lambda\|\alpha\|_{L_{2}(\Omega)}^{2}+\text { DATA. }
\end{aligned}
$$

Assuming sufficient smallness of $\lambda$ and $\|u\|_{L_{\infty}(\Omega)} \leq \varepsilon_{0}$ in terms of the domain only (and in particular independently of $\nu$ which has to be less than a constant, say $\nu_{0}(\Omega, \Gamma)$, which also depends on the domain only), we obtain

$$
\begin{aligned}
\frac{k}{4} \sup _{t} \int_{Y_{t}} \alpha^{2} d \sigma+\nu \int_{\Omega} & |\nabla \alpha|^{2} d x \\
\leq & \frac{k}{8} \sup _{t} \int_{Y_{t}} \alpha^{2} d \sigma+\frac{1}{2} \nu \int_{\Omega}|\nabla \alpha|^{2} d x+\text { DATA. }
\end{aligned}
$$

So we get the main a priori bound

$$
\sup _{t} \int_{Y_{t}} \alpha^{2} d \sigma+\nu \int_{\Omega}|\nabla \alpha|^{2} d x \leq \text { DATA. }
$$

The quantities in DATA are independent of $\nu$ and are sufficiently small. To close the estimation we are required to show that inequality (3.16) implies $\|u\|_{L_{\infty}(\Omega)} \leq \varepsilon_{0}$, but

$$
\begin{array}{ll}
\operatorname{rot} u=\alpha & \text { in } \Omega, \\
\operatorname{div} u=0 & \text { in } \Omega, \\
\vec{n} \cdot u=0 & \text { on } \partial \Omega .
\end{array}
$$

We want to show that solutions to (3.17) belong to $C^{a}(\bar{\Omega})$ for $a<1 / 2$. By $(3.17)_{2}$ we can introduce a stream function $\phi$ such that $u=\nabla^{\perp} \phi$ and

$$
\begin{array}{ll}
\Delta \phi=\alpha & \text { in } \Omega, \\
\phi=0 & \text { in } \partial \Omega .
\end{array}
$$

The estimate (3.16) guarantees that for any $2 \leq p<\infty$ we have

$$
\alpha \in L_{p}\left(t_{\min }, t_{\max } ; L_{2}\left(Y_{t}\right)\right) .
$$

Trivially we have $u=\nabla^{\perp} \phi \in H^{1}(\Omega)$. To obtain the Hölder continuity of $u$ we examine a model problem in the plane

$$
\Delta \psi=\beta \quad \text { in } \mathbb{R}^{2}, \quad \text { where } \beta \in L_{p}\left(\mathbb{R} ; L_{2}(\mathbb{R})\right) .
$$

Then using the Fourier transform we get

$$
\nabla^{2} \psi=\mathcal{F}_{x}^{-1}\left[\frac{\xi \otimes \xi}{|\xi|^{2}}\right] \beta, \quad \text { hence also } \quad \nabla^{2} \psi \in L_{p}\left(\mathbb{R} ; L_{2}(\mathbb{R})\right) .
$$


Then the embedding theorem implies

$$
\|\nabla \psi\|_{C^{a}\left(\mathbb{R}^{2}\right)} \leq C\left(\|\beta\|_{L_{p}\left(\mathbb{R} ; L_{2}(\mathbb{R})\right)}+\|\nabla \psi\|_{H^{1}\left(\mathbb{R}^{2}\right)}\right) \quad \text { for } a<1 / 2-1 / p .
$$

To apply the above result to (3.18) we just solve the equation (3.19) with $\alpha$ extended by zero, using the localization techniques. Then by (3.20), we can remove the inhomogeneity from the r.h.s. of (3.18), getting a standard problem in Hölder spaces, for which we are able to show

$$
u \in C^{a}(\bar{\Omega}) \quad \text { with } a<1 / 2-1 / p .
$$

Finally, we get

$$
\|u\|_{L_{\infty}(\Omega)}+\langle u\rangle_{C^{a}(\bar{\Omega})} \leq C_{p}(\mathrm{DATA}) \leq \varepsilon_{0}
$$

as DATA are sufficiently small and $\langle\cdot\rangle_{C^{a}(\bar{\Omega})}$ denotes the main seminorm in the Hölder space $C^{a}(\bar{\Omega})$. The estimate (3.21) enables us to proceed to the next step in our proof.

Existence. Set

$$
\Xi=\left\{u \in C\left(\bar{\Omega} ; \mathbb{R}^{2}\right): \vec{n} \cdot u=0 \text { and }\|u\|_{L_{\infty}(\Omega)} \leq \varepsilon_{0}\right\} .
$$

Next, we define a map $K: \Xi \rightarrow C\left(\bar{\Omega} ; \mathbb{R}^{2}\right)$ by setting

$$
K(\bar{u})=u,
$$

where $u$ is the solution to the problem

$$
\begin{array}{ll}
\operatorname{rot} u=\alpha & \text { in } \Omega, \\
\operatorname{div} u=0 & \text { in } \Omega, \\
\vec{n} \cdot u=0 & \text { on } \partial \Omega
\end{array}
$$

and $\alpha$ is the solution to

$$
\begin{array}{ll}
\nabla \theta \cdot \nabla \alpha-\nu \Delta \alpha=\operatorname{rot} F-\bar{u} \cdot \nabla \alpha & \text { in } \Omega, \\
\alpha=\alpha_{\text {in }} & \text { on } \Gamma_{\text {in }}, \\
\partial \alpha / \partial \vec{n}=0 & \text { on } \partial \Omega \backslash \Gamma_{\text {in }} .
\end{array}
$$

We see that a fixed point of $K$ defines a solution to system (3.2).

Lemma 3.1. Let $\bar{u} \in \Xi$ and assume that

$$
\|\operatorname{rot} F\|_{L_{2}(\Omega)}+\left\|\alpha_{\mathrm{in}}\right\|_{H^{3 / 2}\left(\Gamma_{\mathrm{in}}\right)} \leq \varepsilon_{1} .
$$

Then $u \in \Xi$, i.e. $K: \Xi \rightarrow \Xi$. Moreover, $K$ is a contraction on $\Xi$. Consequently, there exists a unique fixed point of $K$ belonging to $\Xi$.

Proof. To obtain $u \in \Xi$ it is enough to follow the steps of the a priori bound. To prove that $K$ is a contraction we show that

$$
\left\|K\left(\bar{u}_{1}\right)-K\left(\bar{u}_{2}\right)\right\|_{L_{\infty}(\Omega)} \leq \frac{1}{2}\left\|\bar{u}_{1}-\bar{u}_{2}\right\|_{L_{\infty}(\Omega)},
$$


and

$$
\begin{array}{ll}
\operatorname{rot}\left(u_{1}-u_{2}\right)=\alpha_{1}-\alpha_{2} & \text { in } \Omega, \\
\operatorname{div}\left(u_{1}-u_{2}\right)=0 & \\
\vec{n} \cdot\left(u_{1}-u_{2}\right)=0 & \text { in } \Omega,
\end{array}
$$

where $u_{1}=K\left(\bar{u}_{1}\right), u_{2}=K\left(\bar{u}_{2}\right)$ and $\alpha_{1}, \alpha_{2}$ are the vorticities satisfying (3.23) for $\bar{u}=\bar{u}_{1}$ and $\bar{u}=\bar{u}_{2}$, respectively. The difference $\alpha_{1}-\alpha_{2}$ satisfies

$$
\begin{aligned}
& \nabla \theta \cdot \nabla\left(\alpha_{1}-\alpha_{2}\right)-\nu \Delta\left(\alpha_{1}-\alpha_{2}\right) \\
& =\bar{u}_{1} \cdot \nabla\left(\alpha_{1}-\alpha_{2}\right)+\left(\bar{u}_{1}-\bar{u}_{2}\right) \nabla \alpha_{2} \quad \text { in } \Omega, \\
& \alpha_{1}-\alpha_{2}=0 \quad \text { on } \Gamma_{\text {in }} \text {, } \\
& \frac{\partial}{\partial \vec{n}}\left(\alpha_{1}-\alpha_{2}\right)=0 \quad \text { on } \partial \Omega \backslash \Gamma_{\text {in }} \text {. }
\end{aligned}
$$

Again we repeat the steps (3.3)-(3.21) and get (3.25), provided that the data and $\varepsilon_{0}$ are sufficiently small. Inequality (3.25) implies the existence of a unique fixed point $K(u)=u$, which yields a solution of the system (1.5).

However, the above considerations only apply to small data: at this step we have proven the following.

Proposition 3.1. There exist $\varepsilon_{0}=\varepsilon_{0}(\Omega, \Gamma)$ and $\nu_{0}=\nu_{0}(\Omega, \Gamma)$ such that for any $\alpha_{\mathrm{in}} \in H^{3 / 2}\left(\Gamma_{\mathrm{in}}\right)$, any $F \in H^{1}(\Omega)$ satisfying

$$
\left\|\alpha_{\text {in }}\right\|_{H^{3 / 2}\left(\Gamma_{\text {in }}\right)}+\|F\|_{H^{1}(\Omega)} \leq \varepsilon_{0},
$$

and any $\nu \in\left(0, \nu_{0}\right)$, there exists a solution to the system $(1.5)$ with $d=\bar{d}=$ $\nabla \theta \cdot \vec{n}$ on $\partial \Omega$, and with the estimate (1.9) satisfied independently of $\nu$.

Now let us justify the passage from Proposition 3.1 to Theorem 1. This is a homogeneity argument: if $(u, p)$ satisfies

$$
(u \cdot \nabla) u-\nu \Delta u+\nabla p=F,
$$

then $u^{\varrho}:=\varrho u$ and $p^{\varrho}:=\varrho^{2} p$ satisfy

$$
\left(u^{\varrho} \cdot \nabla\right) u^{\varrho}-\nu \varrho \Delta u^{\varrho}+\nabla p^{\varrho}=\varrho^{2} F .
$$

Now given $\alpha_{\text {in }} \in H^{3 / 2}\left(\Gamma_{\text {in }}\right)$ and $F \in H^{1}(\Omega)$ which do not necessarily satisfy a smallness assumption, we choose $\varrho \leq 1$ small enough in order that

$$
\left\|\varrho \alpha_{\text {in }}\right\|_{H^{3 / 2}\left(\Gamma_{\text {in }}\right)}+\left\|\varrho^{2} F\right\|_{H^{1}(\Omega)} \leq \varepsilon_{0} .
$$

For the data $\left(\varrho \alpha_{\text {in }}, \varrho^{2} F\right)$ one can employ Proposition 3.1, and find a solution for any $\nu \leq \nu_{0}$. Call this solution $\widetilde{u}$. It satisfies in particular $\widetilde{u} \cdot \vec{n}=\bar{d}$ on $\partial \Omega$. 
Now we go back to the original data $\left(\alpha_{\text {in }}, F\right)$, that is, we use the above homogeneity argument with coefficient $1 / \varrho$. We find a solution $u$ of

$$
\begin{array}{ll}
(u \cdot \nabla) u-\frac{\nu}{\varrho} \Delta u+\nabla p=F & \text { in } \Omega, \\
u \cdot \vec{n}=\frac{1}{\varrho} \bar{d} & \text { on } \partial \Omega, \\
\operatorname{rot} u=\alpha_{\text {in }} & \text { on } \Gamma_{\text {in }},
\end{array}
$$

for any $\nu \in\left(0, \nu_{0}\right]$. Hence, we obtain a solution of (1.1) for a wider range of $\nu$, which can include $(0,1]$, by reducing $\varrho$ if necessary.

Now, since $\varrho$ is chosen to satisfy (3.28) and so can be arbitrarily small, the boundary condition $d=(1 / \varrho) \bar{d}$ becomes (1.8) for large enough $l$. That $u$ satisfies (1.9) is a straightforward consequence of the fact that $\widetilde{u}$ satisfies (1.9) (of course, the constant depends on $l$ ). This ends the proof of Theorem 1 .

4. Proof of Theorem 2. A key problem in the passage to the limit is the control of dependence of the inviscid solutions on the boundary data. The main difficulty is related to $\alpha_{\text {in }}$ on $\Gamma_{\text {in }}$, since the vorticity is uniformly bounded in a space which does not control the trace (we may take the $L_{2^{-}}$ space). That is the reason we shall choose a special class of test functions.

For fixed $\nu>0$ the solutions to the Navier-Stokes equations are regular. In particular, we have

$$
\int_{\Omega} v^{\nu} \cdot \nabla \alpha^{\nu} \phi d x-\nu \int_{\Omega} \Delta \alpha^{\nu} \phi d x=\int_{\Omega} \operatorname{rot} F \phi d x
$$

for each $\phi \in C^{\infty}(\bar{\Omega} ; \mathbb{R})$ such that $\left.\phi\right|_{\Gamma_{\text {out }}}=0$.

The choice of $\phi$ makes it possible to take into account the influence of the boundary vorticity $\alpha_{\text {in }}$ on $\Gamma_{\text {in }}$ as well as to neglect information on $\Gamma_{\text {out }}$.

Then (4.1) reads

$$
\begin{aligned}
-\int_{\Omega} v^{\nu} \alpha^{\nu} \nabla \phi d x+\int_{\Gamma_{\mathrm{in}}} \vec{n} \cdot v^{\nu} \alpha_{\mathrm{in}} \phi & d \sigma-\nu \int_{\Gamma_{\mathrm{in}}} \frac{\partial \alpha^{\nu}}{\partial \vec{n}} \phi d \sigma \\
& +\nu \int_{\Omega} \nabla \alpha^{\nu} \nabla \phi d x=\int_{\Omega} \operatorname{rot} F \phi d x .
\end{aligned}
$$

The above equality follows from integration by parts, and the form of the boundary terms is a consequence of the boundary conditions:

$$
\begin{array}{ll}
\vec{n} \cdot v^{\nu}=0 & \text { on } \partial \Omega \backslash\left(\Gamma_{\text {in }} \cup \Gamma_{\text {out }}\right), \\
\phi=0 & \text { on } \Gamma_{\text {out }}, \\
\partial \alpha^{\nu} / \partial \vec{n}=0 & \text { on } \partial \Omega \backslash \Gamma_{\text {in }} .
\end{array}
$$

The estimates proved in Theorem 1 guarantee for $a<1 / 2$ the following 
bound uniformly in $\nu$ :

$$
\left\|v^{\nu}\right\|_{C^{a}(\bar{\Omega})}+\left\|v^{\nu}\right\|_{H^{1}(\Omega)}+\nu^{1 / 2}\left\|\nabla \alpha^{\nu}\right\|_{L_{2}(\Omega)} \leq C .
$$

So for any $\delta>0$ we find a subsequence $\nu_{k} \rightarrow 0^{+}$such that as $k \rightarrow \infty$,

$$
v^{\nu_{k}} \rightarrow v^{E} \quad \text { in } C^{1 / 2-\delta}(\bar{\Omega}) \text { and } \quad v^{\nu_{k}} \rightarrow v^{E} \quad \text { in } H^{1}(\Omega),
$$

for some divergence-free function $v^{E}$.

To examine the limit of (4.2) for this subsequence we analyze the behavior of the third and fourth terms of the 1.h.s. of (4.2). By (4.4) the last term vanishes as $\nu \rightarrow 0$, since

$$
\nu\left|\int_{\Omega} \nabla \alpha^{\nu} \nabla \phi d x\right| \leq \nu^{1 / 2}\left[\nu^{1 / 2}\left\|\nabla \alpha^{\nu}\right\|_{L_{2}(\Omega)}\right]\|\nabla \phi\|_{L_{2}(\Omega)} \leq C \nu^{1 / 2} \rightarrow 0 .
$$

However, the main difficulty is in the third term. To find a good estimate we have to control the normal derivative of $\alpha^{\nu}$. As in the proof of Theorem 1 (see considerations for (3.7)-(3.14)) we obtain ( $\phi$ is given and fixed)

$$
\begin{aligned}
\nu\left|\int_{\Gamma_{\mathrm{in}}} \frac{\partial \alpha^{\nu}}{\partial \vec{n}} \phi d \sigma\right| \leq & C \nu\left\|\frac{\partial \alpha^{\nu}}{\partial \vec{n}}\right\|_{L_{2}\left(\Gamma_{\mathrm{in}}\right)} \\
\leq & C \nu\left\|\nabla \alpha^{\nu}\right\|_{L_{2}(\Omega)}^{1 / 2}\left(\left\|\nabla^{2} \alpha^{\nu}\right\|_{L_{2}(\Omega)}^{1 / 2}+\left\|\nabla \alpha^{\nu}\right\|_{L_{2}(\Omega)}^{1 / 2}\right) \\
\leq & C \nu\left\|\nabla \alpha^{\nu}\right\|_{L_{2}(\Omega)} \\
& +C \nu^{1 / 4}\left[\nu^{1 / 4}\left\|\nabla \alpha^{\nu}\right\|_{L_{2}(\Omega)}^{1 / 2}\right]\left[\nu^{1 / 2}\left\|\nabla^{2} \alpha^{\nu}\right\|_{L_{2}(\Omega)}^{1 / 2}\right] \\
\leq & C\left(\nu^{1 / 2} I_{\nu}+I_{\nu}^{1 / 2} J^{\nu}\right),
\end{aligned}
$$

with $I_{\nu}:=\nu^{1 / 2}\left\|\nabla \alpha^{\nu}\right\|_{L_{2}(\Omega)}$ and $J_{\nu}:=\nu^{1 / 4}\left[\nu^{1 / 2}\left\|\nabla^{2} \alpha^{\nu}\right\|_{L_{2}(\Omega)}^{1 / 2}\right]$. Note that the first term on the r.h.s. of (4.7) satisfies $\nu^{1 / 2} I^{\nu} \rightarrow 0$ by the same arguments as for (4.6). To control the second derivatives, a modification of Theorem 3 is required.

We have

THEOREM 4. Let $G \in L_{2}(\Omega), \beta_{\text {in }} \in H^{3 / 2}\left(\Gamma_{\text {in }}\right)$ and $V \in C^{a}(\bar{\Omega})$ with $a<1 / 2$. Then for $0<\nu \leq 1$ there exists a unique solution to

$$
\begin{array}{ll}
V \cdot \nabla \beta-\nu \Delta \beta=G & \text { in } \Omega, \\
\beta=\beta_{\text {in }} & \text { on } \Gamma_{\text {in }}, \\
\partial \beta / \partial \vec{n}=0 & \text { on } \partial \Omega \backslash \Gamma_{\text {in }},
\end{array}
$$

such that $\beta \in H^{2}(\Omega)$. Additionally, the following estimate is valid:

$$
\begin{aligned}
\nu\left\|\nabla^{2} \beta\right\|_{L_{2}(\Omega)} \leq & C\left[\|G\|_{L_{2}(\Omega)}+\left\|\beta_{\text {in }}\right\|_{H^{3 / 2}\left(\Gamma_{\text {in }}\right)}\right. \\
& \left.+\left(\nu \lambda^{-1}+\lambda^{a}\right)\|\nabla \beta\|_{L_{2}(\Omega)}+\lambda^{a-1}\|\beta\|_{L_{2}(\Omega)}\right],
\end{aligned}
$$

where $\lambda$ with $0<\lambda \leq \lambda_{0}$ is a localization parameter and the constant $C$ is independent of $\lambda$ and $\nu$, and depends only on the shape of $\partial \Omega$. 
Applying (4.9) to $J^{\nu}$, from (4.7) we deduce that

$$
\begin{aligned}
J^{\nu} \leq & C \nu^{1 / 4}\left[\|\operatorname{rot} F\|_{L_{2}(\Omega)}+\left\|\alpha_{\text {in }}\right\|_{H^{3 / 2}\left(\Gamma_{\text {in }}\right)}\right. \\
& \left.+\left(\nu \lambda^{-1}+\lambda^{a}\right)\left\|\nabla \alpha^{\nu}\right\|_{L_{2}(\Omega)}+\lambda^{a-1}\left\|\alpha^{\nu}\right\|_{L_{2}(\Omega)}\right]^{1 / 2} \\
\leq & \kappa(\nu)+C\left[\left(\nu \lambda^{-1}+\lambda^{a}\right) \nu^{1 / 2}\left\|\nabla \alpha^{\nu}\right\|_{L_{2}(\Omega)}+\nu^{1 / 2} \lambda^{a-1}\left\|\alpha^{\nu}\right\|_{L_{2}(\Omega)}\right]^{1 / 2},
\end{aligned}
$$

where $\kappa(\nu) \rightarrow 0$ as $\nu \rightarrow 0$.

Keeping in mind (4.4) we describe relations between $\nu$ and $\lambda$. This is reasonable since the constant in (4.9) is independent of $\nu$ and $\lambda$. Taking

$$
\nu \leq \lambda^{2} \leq 2 \nu \leq 2
$$

we obtain

$$
J^{\nu} \leq \kappa(\nu)+C\left[\nu^{1 / 2}+\nu^{a / 2}+\nu^{a / 2}\right]^{1 / 2} \rightarrow 0 .
$$

So the limit of (4.2) reads

$$
-\int_{\Omega} v^{E} \alpha^{E} \cdot \nabla \phi d x+\int_{\Gamma_{\mathrm{in}}} \vec{n} \cdot v^{E} \alpha_{\mathrm{in}} \phi d \sigma=\int_{\Omega} \operatorname{rot} F \phi d x
$$

for $\phi \in C^{\infty}(\bar{\Omega})$ with $\left.\phi\right|_{\Gamma_{\text {out }}}=0$.

Thanks to the choice of the test functions we obtain a dependence on the boundary vorticity $\alpha_{\text {in }}$. Additionally the above integral identity is a weak formulation of the Euler system (1.11). Theorem 2 is proved.

5. Appendix. Here we prove Theorems 3 and 4.

Proof of Theorem 3. By the assumptions we can find an extension of $\beta_{\text {in }}$ such that

$$
\widetilde{\beta} \in H^{2}(\Omega),\left.\quad \frac{\partial \widetilde{\beta}}{\partial \vec{n}}\right|_{\partial \Omega}=0,\left.\quad \widetilde{\beta}\right|_{\Gamma_{\mathrm{in}}}=\beta_{\mathrm{in}} .
$$

Then we set $\beta_{\text {new }}=\beta_{\text {old }}-\widetilde{\beta}$, getting (3.10) with the homogeneous condition: $\left.\beta\right|_{\Gamma_{\mathrm{in}}}=0$ and

$$
G_{\text {new }}=G_{\text {old }}+\nu \Delta \widetilde{\beta}+\nabla \theta \cdot \nabla \widetilde{\beta}
$$

with a suitable estimate in the $L_{2}$-space.

Now we start with local estimates. Consider a smooth partition of unity $\left\{\pi_{k}\right\}_{k \in I}$ such that

$$
\pi_{k}: \Omega \rightarrow[0,1] \text { and } \sum_{k \in I} \pi_{k} \equiv 1 \quad \text { on } \Omega .
$$

Additionally, we suppose that $I=\mathcal{N} \cup \mathcal{I}$, the summands are finite sets, and

$$
\operatorname{supp} \pi_{k} \cap \partial \Omega \neq \emptyset \quad \text { for } k \in \mathcal{N}, \quad \operatorname{supp} \pi_{k} \cap \partial \Omega=\emptyset \quad \text { for } k \in \mathcal{I} ;
$$


moreover

$$
\sup _{k} \operatorname{diam}\left(\operatorname{supp} \pi_{k}\right) \leq \lambda, \quad\left|\nabla \pi_{k}\right| \leq C / \lambda, \quad\left|\nabla^{2} \pi_{k}\right| \leq C / \lambda^{2}
$$

and the Lebesgue number of the cover $\left\{\operatorname{supp} \pi_{k}\right\}$, denoted by $N_{0}$, does not increase for all $0<\lambda \leq \lambda_{0}$. It depends only on the regularity of the boundary $\partial \Omega$. In our considerations we choose $\left\{\pi_{k}\right\}_{k \in I}$ with sufficiently small $\lambda$.

The interior estimate. Applying $\pi_{k}$ with $k \in \mathcal{I}$ to the system (3.10) we obtain

$$
\overline{\nabla \theta} \cdot \nabla\left(\pi_{k} \beta\right)-\nu \Delta\left(\pi_{k} \beta\right)=\pi G+R_{1} \quad \text { in } \mathbb{R}^{2},
$$

where

$$
R_{1}=2 \nu \nabla \pi_{k} \cdot \nabla \beta+\nu\left(\Delta \pi_{k}\right) \beta+(\overline{\nabla \theta}-\nabla \theta) \pi_{k} \nabla \beta+(\overline{\nabla \theta}-\nabla \theta) \beta \nabla \pi_{k}
$$

with $\overline{\nabla \theta}=\nabla \theta\left(x_{k}\right)$ and $x_{k} \in \operatorname{int} \operatorname{supp} \pi_{k}$.

The symbol of the operator on the l.h.s. of (5.3) has the form

$$
i \overline{\nabla \theta} \cdot \xi+\nu|\xi|^{2}
$$

and in particular satisfies

$$
\left.\left.|i \overline{\nabla \theta} \cdot \xi+\nu| \xi\right|^{2}|\geq \nu| \xi\right|^{2} .
$$

A direct application of Parseval's identity and the definition of $H^{m}$ together with (5.5) lead straightforwardly to the estimate

$$
\nu\left\|\nabla^{2}\left(\pi_{k} \beta\right)\right\|_{L_{2}\left(O_{k}\right)} \leq C\left(\left\|\pi_{k} G\right\|_{L_{2}\left(O_{k}\right)}+\left\|R_{1}\right\|_{L_{2}\left(O_{k}\right)}\right),
$$

where $O_{k}=\operatorname{supp} \pi_{k}$ and by (5.2) and (5.4) we find

$$
\begin{aligned}
& \left\|R_{1}\right\|_{L_{2}\left(O_{k}\right)} \\
& \leq C\left[\nu \lambda^{-1}\|\nabla \beta\|_{L_{2}\left(O_{k}\right)}+\nu \lambda^{-2}\|\beta\|_{L_{2}\left(O_{k}\right)}+\lambda\|\nabla \beta\|_{L_{2}\left(O_{k}\right)}+\|\beta\|_{L_{2}\left(O_{k}\right)}\right] .
\end{aligned}
$$

where the constant in (5.7) is independent of $\nu$ and $\lambda$.

The boundary estimate. Applying $\pi_{k}$ with $k \in \mathcal{N}$ to the system (3.10) we obtain

$$
\begin{array}{ll}
\overline{\nabla \theta} \cdot \nabla\left(\pi_{k} \beta\right)-\nu \Delta\left(\pi_{k} \beta\right)=\pi G+R_{1} & \text { in } \Omega, \\
\frac{\partial\left(\pi_{k} \beta\right)}{\partial \vec{n}}=0 \quad \text { and } / \text { or } \quad \pi_{k} \beta=0 & \text { on } \partial \Omega \cap O_{k},
\end{array}
$$

where $R_{1}$ is given by (5.4) and $\overline{\nabla \theta}=\nabla \theta\left(x_{k}\right)$, but with $x_{k} \in \operatorname{int} \operatorname{supp} \pi_{k} \cap \partial \Omega$. The boundary conditions depend on the localization of the support of $\pi_{k}$ with respect to the localization of $\Gamma_{\mathrm{in}}$.

The smoothness of the boundary allows us to transport this system onto the halfspace $\mathbb{R}_{+}^{2}$ with a local coordinate system $\left(z_{1}, z_{2}\right)$. For each $\pi_{k}$ we 
consider a map $Z_{k}: \Omega \cap O_{k} \rightarrow \mathbb{R}_{+}^{2}$. Then equations (5.8) read

$$
\begin{aligned}
& \overline{\nabla \theta} \cdot \nabla_{z} Z_{k}^{-1 *}(\pi \beta)-\nu \Delta_{z} Z_{k}^{-1 *}(\pi \beta) \\
& =Z_{k}^{-1 *}(\pi G)+Z_{k}^{-1 *}\left(R_{1}\right)+R_{2} \quad \text { in } \mathbb{R}_{+}^{2}, \\
& \frac{\partial Z_{k}^{-1 *}\left(\pi_{k} \beta\right)}{\partial z_{2}}=0 \quad \text { and } / \text { or } \quad Z_{k}^{-1 *}\left(\pi_{k} \beta\right)=0 \quad \text { on } \mathbb{R} \times\{0\},
\end{aligned}
$$

where

$$
R_{2}=\bar{\nabla} \theta\left(\nabla_{x}-\nabla_{z}\right) Z_{k}^{-1 *}\left(\pi_{k} \beta\right)+\nu\left(\Delta_{x}-\Delta_{z}\right) Z_{k}^{-1 *}\left(\pi_{k} \beta\right),
$$

$\nabla_{z}$ denotes the gradient in $\mathbb{R}^{2}$ in the $z$-coordinates and $\nabla_{x}$ denotes the gradient in the $x$-coordinates transformed by $Z_{k}$.

The above problem reduces to a model problem with three possibilities. We have to consider the equation

$$
\overline{\nabla \theta} \cdot \nabla \gamma-\nu \Delta \gamma=H \quad \text { in } \mathbb{R}_{+}^{2},
$$

with three types of boundary relations

(i) $\partial \gamma / \partial \vec{n}=0$ on $\mathbb{R} \times\{0\}$;

(ii) $\gamma=0$ on $\mathbb{R} \times\{0\}$;

(iii) $\partial \gamma / \partial \vec{n}=0$ for $z_{1}<0$ and $\gamma=0$ for $z_{1} \geq 0$ on $\mathbb{R} \times\{0\}$.

We need the following bound on the solutions to (3.10):

$$
\nu\left\|\nabla^{2} \gamma\right\|_{L_{2}\left(\mathbb{R}_{+}^{2}\right)} \leq C\|H\|_{L_{2}\left(\mathbb{R}_{+}^{2}\right)} .
$$

The first two cases follow from the standard approach. Case (i) uses the method of symmetry to transform the system into the whole space. Case (ii) requires the standard energy estimate. Case (iii) is not straightforward, because of the structure of the boundary conditions. Here we have to specify the choice of the point $x_{k}$. If $\partial \Gamma_{\text {in }} \in \operatorname{int} \operatorname{supp} \pi_{k}$ then we choose $x_{k}$ as the end of $\Gamma_{\text {in }}$ (see (5.4)). This choice implies that $\overline{\nabla \theta} \perp \vec{n}$ on $\partial \Gamma_{\text {in }}$ and this form of (5.11) allows us to apply the standard energy method to get the estimate (5.13). It is enough to test the equation with $\gamma_{z_{1}}, \gamma_{z_{2} z_{2}}$.

Thus the bound used for (5.11) yields the following estimate for (5.9):

$$
\begin{aligned}
\nu\left\|\nabla^{2} Z_{k}^{-1 *}\left(\pi_{k} \beta\right)\right\|_{L_{2}\left(\mathbb{R}_{+}^{2}\right) \leq} & C\left[\left\|Z_{k}^{-1 *}\left(\pi_{k} G\right)\right\|_{L_{2}\left(\mathbb{R}_{+}^{2}\right)}\right. \\
& \left.+\left\|Z_{k}^{-1 *}\left(R_{1}\right)\right\|_{L_{2}\left(\mathbb{R}_{+}^{2}\right)}+\left\|R_{2}\right\|_{L_{2}\left(\mathbb{R}_{+}^{2}\right)}\right]
\end{aligned}
$$

with (in view of regularity of the boundary, i.e. regularity of $Z_{k}$ )

$$
\begin{aligned}
\left\|R_{2}\right\| \leq & C\left(\lambda\left\|\nabla_{z} Z_{k}^{-1 *}\left(\pi_{k} \beta\right)\right\|_{L_{2}\left(\mathbb{R}_{+}^{2}\right)}\right. \\
& \left.+\nu \lambda\left\|\nabla_{z}^{2} Z_{k}^{-1 *}\left(\pi_{k} \beta\right)\right\|_{L_{2}\left(\mathbb{R}_{+}^{2}\right)}+\nu\left\|\nabla_{z} Z_{k}^{-1 *}\left(\pi_{k} \beta\right)\right\|_{L_{2}\left(\mathbb{R}_{+}^{2}\right)}\right),
\end{aligned}
$$

and the constants in (5.14) and (5.15) are independent of $\nu$ and $\lambda$. Now we first apply Poincaré's inequality to the last term on the r.h.s.: this allows us 
to include it in the second one. And at this point we require the parameter $\lambda$ to be so small that the last two terms can be put on the l.h.s. of (5.14).

So for $k \in \mathcal{N}$ we have

$$
\begin{aligned}
& \nu\left\|\nabla^{2}\left(\pi_{k} \beta\right)\right\|_{L_{2}\left(O_{k}\right)} \\
& \quad \leq C\left(\lambda\|\nabla \beta\|_{L_{2}\left(O_{k}\right)}+\|\beta\|_{L_{2}\left(O_{k}\right)}+\left\|\pi_{k} G\right\|_{L_{2}\left(O_{k}\right)}+\left\|R_{1}\right\|_{L_{2}\left(O_{k}\right)}\right),
\end{aligned}
$$

but now $C$ in (5.16) depends on $Z_{k}$, so on the regularity of $\partial \Omega$.

Summing up (5.6) and (5.16), noting that

$$
\nu^{2}\left\|\nabla^{2} \beta\right\|_{L_{2}(\Omega)}^{2} \leq \nu^{2} N_{0} \sum_{k}\left\|\nabla^{k}\left(\pi_{k} \beta\right)\right\|_{L_{2}(\Omega)}^{2},
$$

since the Lebesgue number $N_{0}$ is independent of the smallness of the localization parameter $\lambda$, we get (3.11). In particular, we can use it in considerations for the limit $\lambda \rightarrow 0$.

Theorem 3 has been proved.

Proof of Theorem 4. We present here only the main difference between the proofs of Theorems 3 and 4. Since for (4.8) the vector field $V$ is in $C^{a}(\bar{\Omega})$ only, we look closer at the estimate of $R_{1}$ (see (5.4)). Pointing out the difference we have

$$
\begin{aligned}
\left\|(\bar{V}-V) \pi_{k} \nabla \beta\right\|_{L_{2}\left(O_{k}\right)}+ & \left\|(\bar{V}-V) \beta \nabla \pi_{k}\right\|_{L_{2}\left(O_{k}\right)} \\
& \leq C \lambda^{a}\|\nabla \beta\|_{L_{2}\left(O_{k}\right)}+C \lambda^{a-1}\|\beta\|_{L_{2}\left(O_{k}\right)} .
\end{aligned}
$$

The rest of the estimation is the same since the other assumptions are identical. This way we prove (4.9) and Theorem 4.

Acknowledgments. The first author is partially supported by grant JCJC06_137283 of the Agence Nationale de la Recherche. The second author has been supported by Polish KBN grant No. 1 P03A 02130 and by ECFP6 M.Curie ToK program SPADE2, MTKD-CT-2004-014508 and SPB-M. The second author thanks Laboratoire Jacques-Louis Lions for their hospitality, where the research was performed.

\section{References}

[1] R. W. Brockett, Asymptotic stability and feedback stabilization, in: Differential Geometric Control Theory (Houghton, MI, 1982), Progr. Math. 27, Birkhäuser Boston, 1983, 181-191.

[2] J.-M. Coron, Contrôlabilité exacte frontière de l'équation d'Euler des fluides parfaits incompressibles bidimensionnels, C. R. Acad. Sci. Paris Sér. I Math. 317 (1993), 271-276.

[3] - Sur la stabilisation des fluides parfaits incompressibles bidimensionnels, in: Séminaire Équations aux Dérivées Partielles, 1998-1999, exp. VII, École Polytech., Palaiseau, 1999, 17 pp. 
[4] J.-M. Coron, On the null asymptotic stabilization of 2-D incompressible Euler equation in a simply connected domain, SIAM J. Control Optim. 37 (1999), 1874-1896.

[5] O. Glass, Existence of solutions for the two-dimensional stationary Euler system for ideal fluids with arbitrary force, Ann. Inst. H. Poincaré Anal. Non Linéaire 20 (2003), 921-946.

[6] - Asymptotic stabilizability by stationary feedback of the two-dimensional Euler equation: the multiconnected case, SIAM J. Control Optim. 44 (2005), 1105-1147.

[7] P. B. Mucha, The Eulerian limit and the slip boundary conditions-admissible irregularity of the boundary, in: Regularity and Other Aspects of the Navier-Stokes Equations, Banach Center Publ. 70, Inst. Math., Polish Acad. Sci., Warszawa, 2005, $169-183$.

[8] - On the inviscid limit of the Navier-Stokes equations for flows with large flux, Nonlinearity 16 (2003), 1715-1732.

[9] P. B. Mucha and M. Pokorný, On a new approach to the issue of existence and regularity for the steady compressible Navier-Stokes equations, Nonlinearity 19 (2006), 1747-1768.

[10] C. Pommerenke, Boundary Behaviour of Conformal Maps, Grundlehren Math. Wiss. 299, Springer, Berlin, 1992.

[11] H. Triebel, Spaces of Besov-Hardy-Sobolev Type, Teubner Verlagsgesellschaft, Leipzig, 1978.

[12] W. M. Zajączkowski, Local solvability of a nonstationary leakage problem for an ideal incompressible fluid. III, Math. Methods Appl. Sci. 4 (1982), 1-14.

[13] - Solvability of an initial-boundary value problem for the Euler equations in twodimensional domain with corners, ibid. 6 (1984), 1-22.

[14] —, Some leakage problems for ideal incompressible fluid motion in domains with edges, in: Partial Differential Equations (Warszawa, 1984), Banach Center Publ. 19, PWN, Warszawa, 1987, 383-397.

UMR 7598

Laboratoire Jacques-Louis Lions

CNRS et UPMC Paris 6

F-75005 Paris, France

E-mail: glass@ann.jussieu.fr
Instytut Matematyki Stosowanej i Mechaniki

Uniwersytet Warszawski

Banacha 2

02-097 Warszawa, Poland

E-mail: p.mucha@mimuw.edu.pl 
